\title{
The Effect of a Ground-Water Rehabilitation Program on the Speed of Returning to NormalState after Tennis Elbow Injury
}

\author{
AlaaEldin Mohamed Elewa*, Yasser Kamal Ghoniem**, Mohamed Kamal Moussa***
}

This research was conducted to identify the effect of a ground-water rehabilitation program on the speed of returning to normal state after tennis elbow injury. The research was applied on an intentional sample of 11 Egyptian tennis players who are injured with tennis elbow in the time of conducting this research where the diagnosis and injury reports where conducted through a clinical physician. The sample had the medians of age 19.50 years (+/- 0.85$)$, weight $66.50 \mathrm{Kg}(+/-1.08)$ and length $164.44 \mathrm{~cm}(+/-1.56 \mathrm{~cm})$. A questionnaire was distributed among the sample society to determine the exact symptoms of tennis elbow injury where it was proved reliable and subjective. The recommended program was applied on the sample society for 6 weeks, 3 training sessions per week. Pre and post measurements were taken by measuring the range of motion for the injured joint. The results showed the presence of significant differences between the pre and post measurement for the range of motion for the elbow joint in favor of the post measurement a all of the members of the sample society (pre: $70.45+/-1.05$ - post: $115.06+/-0.34$ ) which proves the advantages of using a water-ground rehabilitation program to speed up returning to the normal state after tennis elbow injuries.

Keywords:tennis elbow - injury - rehabilitation - water - ground-physiotherapy.

\section{Introduction}

$\mathbf{R}$ epetitive strain injuries, or overuse injuries of tendons and muscles, are very common and are both treatable and preventable. The severity of these injuries is directly related to the number of repetitions of a particular activity and the technique utilized while performing that activity. The severity is also proportional to the strength of the muscles utilized as compared to the force of the activity.

Edell (2011) stated that Tennis Elbow (Lateral Epicondylitis) is a common cause of elbow pain. Tennis Elbow is characterized by pain over the outer side of the elbow, which may

\footnotetext{
*Professor, Biological Science and Sport Health Department, , The Faculty of Physical Education, Abu Qir, Alexandria University, Egypt.

**Assistant Professor, Sports Games Training Department, , The Faculty of Physical Education, Abu Qir, Alexandria University, Egypt.

***Lecturer, Biological Science and Sport Health Department, , The Faculty of Physical Education, Abu Qir, Alexandria University, Egypt.
}

radiate down the forearm. Despite the name, Tennis Elbow does not just afflict tennis professionals. In fact, this common elbow problem (it affects about 3\% of the population) is most often associated with work-related activities, although racquet sports players are more prone to the condition and account for approximately $5 \%$ of all sufferers. Tennis Elbow is equally common in men and women, peaking in prevalence between the ages of 30 and 50. (8)

Cyriax (2003) stated that The evidence is overwhelmingly in favor of a typical tennis elbow's being caused primarily by a tear between the tendinous origin of the extensor carpi radialisbrevis and the periosteum on the anterior surface of the lateral epicondyle. Secondarily, the continual jerks given to this area of acute traumatic inflammation by muscular contractions set up a chronic periostitis here, and to this the symptoms are referable. The acute periostitis occasioned by a 
direct injury to the epicondyle can become chronic for the same reason, notwithstanding the absence of a tear. Inflammation of the subcutaneous epicondylar bursa and of the radiohumeral bursa is an uncommonbut welldefined entity capable of causing the symptoms of a tennis elbow. Ruptures of a muscle belly are very rare and differ neither in pathology nor in treatment from the same condition elsewhere. The possibility of a nipped synovial fringe's being at fault has yet to be proved. (11)

It has been proved in several researches that rehabilitation programs either inside water or on ground using some tools are very helpful in completing the treatment protocol after injury using electrotherapy, contrast bath, ultrasonic therapy ....etc. $(13: 14)$

Wang et al (2002) and Milne (2008) also stated that cold compression and ice massage is used during rehabilitation for pain relief especially when the patient is practicing some rehabilitation training exercises. Also cold they might help in moving any blood infiltration in the joint tissues. (6:42) (7)

Ali Elbek et al (1994) and AlaaEliwa (1997) agreed that massage is very useful for blood flow and pain relief and improves the muscle tone in the injured organ as it relaxes the connection between bone and muscle where iced massage can be used in several rehabilitation stages. $(4: 67)(3: 25)$

Magima (2002) also pointed out that elbow tendons injuries are considered to be one of the most common injuries as most normal life movements and activities depends on it. Injury mostly occurs due to repetitive movements that causes extra load on cartilage, tendons, ligaments and muscles which causes a conflict in the movement between ulna and radius that leads to chronic inflammation or tissues damage. (16:72)

Alawaldly (2004) mentioned that the medial node pain is very common nowadays as it affects the physical efficiency which in turn affects production and achievements. (1:108)

It is concluded from previous researches and scientific articles that the mechanism of pain in elbow takes place when the joint that lies between two bones is placed in a position of a strong strain in ligaments and tendons in addition to the soft tissues that surround the joint due to the heavy load of repetitive usage of the joint doing the same action for a long time.

Shmidt (2006) stated that when the joint suffers from inflammation, this might cause medium intensity pain but still very annoying. This pain takes place due to the repetitive torsion of the ligament of the extensor muscle of the arm either in sports activities or in normal day activities. It has been proved also that $50 \%$ of sportsmen suffer from elbow injuries. (19: 927, 928)

Avela et al (2002) confirmed the importance of flexibility and stretching exercises in learning and mastering the new techniques and skills as well as preventing injuries. $(5: 24,452)$

Bisset et al (2009) stated that Lateral pain in the elbow affects up to $3 \%$ of the population, and is considered an overload injury of the extensor tendons of the forearm where they attach at the lateral epicondyle. Although usually selflimiting, symptoms may persist for over 1 year in up to $20 \%$ of people (15)

Tennis elbow as an injury has been defined and described in various terms including lateral elbow pain, lateral epicondylitis, lateral epicondylalgia, tendonitis of the common extensor origin, and peritendinitis of the elbow. Tennis elbow is characterised by pain and tenderness over the lateral epicondyle of the humerus, and pain on resisted dorsiflexion of the wrist, middle finger, or both. For the purposes of this review, tennis elbow is restricted to lateral elbow pain or lateral epicondylitis or lateral epicondylalgia. 
Heshmat and Shalaby (2003) mentioned that the constant muscular contraction causes tension in the muscle but without any elongation where no movement occurs in the joint and in this case force equals resistance. (10:85)

Lately water therapy has been used in treating a wide range of injuries due to its quick and remarkable effect in healing. Water therapy programs usually contains walking, running, jumping and stretching inside water where it is $100 \%$ safe to apply in early stages of rehabilitation as it helps is keeping fit and decreases the pressure on the injured tissues. $(14: 148)$

Werner (2005) states that compression of the injured joint has some disadvantages due to lack of movement including analytical changes in the muscular-skeletal system as general weakness in the surrounding muscles. (25: 392)

\section{Tennis elbow injury mechanism:}

Tennis elbow is one of the commonest lesions of the arm and was first described by Runge in
1873. Typically the patient complains of lateral elbow pain that is aggravated by gripping. (20)

Tennis Elbow usually begins as inflammation of the extensor tendons of the forearm as they attach to the Humerus (upper arm) bone, just above the elbow joint. Typically, this inflammation is caused by prolonged gripping activities such as hammering, screwdriving, weightlifting, playing certain musical instruments, canoeing, digging in the garden, driving and, of course, racquet sports and mainly tennis through performing different strokes like forehand, back hand, serve, smash, volleys...etc.

The exact cause of tennis elbow is not known, but it does tend to occur after repetitive use of the extensor tendons at the elbow, originating from the Extensor Carpi Radialis (ECRB) tendon, such as in tennis (hence the name). These tendons are involved in extending the wrist joint and are also active when gripping with the wrist extended (as in tennis). Any activity that twists and extends the wrist can lead to tennis elbow. (19)

Fig (1):

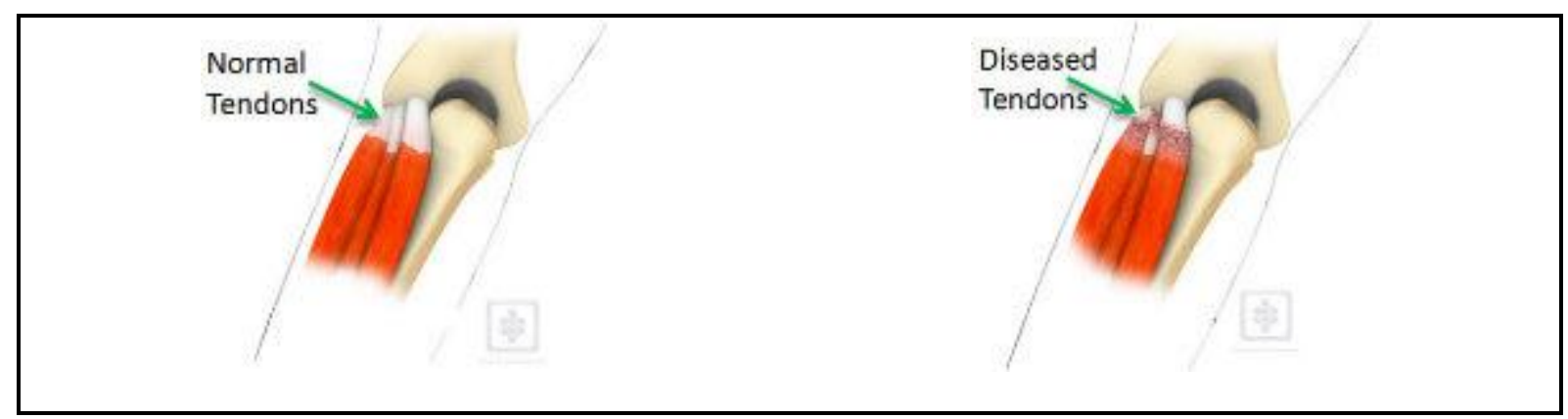

The clinical difference between injured and non injured elbow

\section{Tennis Elbow Signs \& Symptoms}

Tennis Elbow is very easy to diagnose. There is pain when the Lateral Epicondyle (outermost part of the elbow) is touched, and also if the elbow is straight and the hand is moved forward and back at the wrist. The elbow pain is made worse by gripping activities as previously described and, in some cases, simple things like turning a door handle can cause intense pain. X- rays and blood tests can rule out arthritis if the diagnosis is unclear.

It is also characterized by pain on the outside of the elbow and is a problem with tendons around the elbow as well. Tendons attach muscles to bone so using and stretching the muscles will have an effect on the tendon. One or more tendons which are attached around the elbow and help moving the wrist and forearm become inflamed. If the inflammation process continues 
the tendons can then show changes of 'wear and tear', with the tendon tissue becoming thickened or swollen. (21)

\section{Aim of the research}

This researches aims for identifying the effect of a ground-water rehabilitation program on the speed of returning to normal state after tennis elbow injury

Research procedures:

\section{1- Research method:}

The researcher used the experimental method with pre and post measurement on one group.

\section{2- Research sample:}

The study was conducted on 11 tennis players suffering from tennis elbow injury that were chosen intentionally then diagnosed clinically to give a full medical report about the injury through a specialized clinical physician.

Table (1)

\begin{tabular}{|c|c|c|c|}
\hline Variables & Arithmetic mean & Standard deviation & Skewness \\
\hline Range of motion & 70.54 & 1.05 & -0.80 \\
\hline Degree of pain & 6.99 & 0.84 & -0.28 \\
\hline Grip strength for injured limb & 31.19 & 0.24 & 1.756 \\
\hline
\end{tabular}

The statistical information for the research variables among the sample society where the Skewness values are between -/+3 which proves the homogenous choice for the research sample

\section{3- Tools and measurements:}

- Pain degree determination questionnaire that was used to identify the history of injury for the sample society as well as the symptoms and the degree of pain.

- Restameter that was used to measure the height of the sample society for proving the homogenous formation and for applying safety in the water program as well as measuring the length of theraband and elastic ropes to estimate the amount of resistance on the injured limb

- Weight scale to measure the body weight for the sample society

- Goniometer to measure the range of motion for the injures joint

- Dynamometer to measure the muscular strength for the muscle groups of the injured limb.

- Air and metal dumbbells to apply resistance on the injured limb inside water and on ground
- Multi-resistance elastic ropes and theraband to apply resistance on the injured limb inside water

- Ice bags for massaging and compression

- Stop watch for measuring the time of performing the recommended program.

\section{4- The recommended water-ground rehabilitation program:}

The program includes using ice bags and ice cubes for massaging the injured limb for 5-10 minutes before and after applying the exercise program. Afterwards water exercise program is applied after a period of 30 minutes rest bearing in mind the gradual increase in training load over the 2 weeks of each stage in the rehabilitation program.

The water rehabilitation program was based on the following elements as a therapeutic protocol:

1- The hot-cold water contrast using the range of temperatures between 15 and 34 degree centigrade. 
2- Using the multi-resistance theraband and elastic ropes to apply resistance on the injured limb along with the resistance of water to help speed the healing process.

3- Air dumbbells were used inside water to apply resistance on the injured limb imitating the effect of the normal dumbbells exercise on ground.

\section{The stages of the rehabilitation program:}

$1^{\text {st }}$ stage: lasts for 2 weeks, 3 sessions per week were water and ground exercises were applied for developing static muscular strength, stretching and flexibility for elbow joint using air dumbbells, theraband and elastic ropes.

$2^{\text {nd }}$ stage: lasts for 2 weeks, 3 sessions per week including an exercise program to develop dynamic muscular strength using light

dumbbells $(0.5 \mathrm{~kg})$ in addition to positive flexibility and stretching exercises to increase the range of motion for elbow joint. The real playing technique was simulated inside the water using theraband and elastic ropes.

$\mathbf{3}^{\text {rd }}$ stage: lasts for 2 weeks, 3 sessions per week including exercises program to develop and increase muscular strength using light dumbbells $(1 \mathrm{~kg})$ in addition to flexibility and stretching exercises to increase the range of motion for the injured elbow and retain its normal state. The real playing technique was simulated inside the water using theraband and elastic ropes.

Pre and post measurements were applied in same circumstances and in the same order.

\section{Results:}

Table (2)

\begin{tabular}{|c|c|c|c|c|c|}
\hline \multirow{2}{*}{ Variables } & \multicolumn{2}{|c|}{ Pre measurement } & \multicolumn{2}{c|}{ Post measurement } & \multirow{2}{*}{ T } \\
\cline { 2 - 5 } & Mean & SD & Mean & SD & \\
\hline Range of motion & 70.54 & 1,05 & 115.06 & 0.34 & 46.17 \\
\hline
\end{tabular}

The significance of differences between pre and post measurements for the range of motion of elbow joint.

Table (3)

\begin{tabular}{|c|c|c|c|c|c|}
\hline \multirow{2}{*}{ Variables } & \multicolumn{2}{|c|}{ Pre measurement } & \multicolumn{2}{c|}{ Post measurement } & \multirow{2}{*}{ T } \\
\cline { 2 - 5 } & Mean & SD & Mean & SD & \\
\hline Grip strength (kg) & 31.91 & 0.24 & 44.35 & 0.48 & 58.19 \\
\hline Degree of pain & 6.99 & 0.84 & 1.45 & 0.51 & 16.88 \\
\hline
\end{tabular}

The significance of differences between pre and post measurement for the grip strength and degree of pain for the injured limb.

\section{Discussion:}

Table (3) shows the presence of significant differences between the pre and post measurements in the rage of motion for the elbow joint in favor of the post measurements which proves the role of the recommended rehabilitation program in retaining the normal state of the joint for the members of the research sample. The researchers explain this improvement as a result of sticking to the recommended program and performing the exercises with full power in order to develop the flexibility and the strength of the muscles attached to the elbow joint.
Table (4) shows the presence of significant differences between the pre and post measurements for the remaining two research variables which are grip strength and degree of pain in favor of the post measurement which proves the positive effect of the proposed rehabilitation protocol in this research.

It has been also proved that eccentric gradual exercises for elbow flexors and extensors works on increasing the range of motion of the elbow joint. Also Abdelfatah and Nasreldin (1993) proved that gradual endurance exercises works on pain relief and increasing the range of motion for the injured joint. (2) 
UsamaRiad (2001) confirmed that using ice massaging and cold compression helps in decreasing muscle tension and pain relief in addition to helping in decreasing blood infiltration that helps in returning to the normal state quickly.(21)

The research results agrees with Majima's research (2002) and the findings of (2004) that the rehabilitation program should start as soon as the injury takes place as this might help in restoring the rage of motion and flexibility as soon as possible. $(14-17)$

Jeng (2011) also stated that the tennis elbow rehabilitation program has to include stretches, strengthening exercises and advise on modification of technique - either at work or in sport. Sometimes, a sports injury professional will advise the wearing of a brace or support to protect the tendon whilst healing and strengthening. (11)

The speed of returning to the normal state for the sample society can be referred to using several therapeutic protocols including using the ice compression and ice massage as well as the hot-cold contrast bath through controlling the water temperature. Also using the air dumbbells helped in restoring the normal range of motion and the final disposal of pain and inflammation.

\section{Conclusions:}

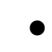

The recommended rehabilitation program had a positive effect on increasing the range of motion for the elbow joint as well as decreasing the degree of pain.

- Using ice and cold compression as a recovery tool had a positive effect on decreasing the degree of inflammation and degree of pain which helped in increasing the muscular strength and the elbow joint range of motion which was shown in the results of the research variables in the post measurement.

- $\quad$ The recommended program included a group of concentric strength exercises and static flexibility exercises to increase muscular strength and the range of motion in addition to using ice after the end of each training session.
Water exercises were more efficient in treating and rehabilitation of the injured tendons and ligaments in the elbow joint.

\section{Recommendations:}

In view of the research results and conclusions, the researchers recommend the following:

1 -

Using the water-ground exercises as a major component in the rehabilitation protocol of tennis elbow injury

2-

Using the recommended exercise program as a preventative tool from tennis elbow injury after the treatment is done.

$3-$

Perform a regular medical follow-up during the rehabilitation program to monitor the changes and to guarantee the safety for the injured players.

$4-$

Further researches should be performed to design preventative programs for similar types of injuries.

\section{References:}

1. Abd El-Azeem A.: The new trends in physiotherapy and sports injuries (Dar ElFekr El Araby - Cairo, 2004)

2. AbouelEla A. Fatah, Ahmed N.: The physiology of physical fitness (Dar El-Fekr El Araby - Cairo, 1993)

3. AlaaEliwa: Health in the sports field (Dar EL-Maaref, Alexandria, 1997)

\section{Ali Al-Bek, HishamMoheeb\&AlaaEliwa:} The sportsman rest (Dar El-Maaref, Alexandria, 1994)

5. Avella et al.: Reducing the risk of injury due to exercise (C BMJ, 451 - 452, 2004)

6. Ching - Jen Wang \& Han Shiang: Shock wave therapy for patients with Lateral Epicondylitis of the Elbow. (The American Journal of Sports Medicine $30: 422-425$, 2002)

7. Chris Milne: Patient Information Sheet for tennis elbow, May 2008 
8. David Edell: The sports medicine specialists, Houston Orthopædics\& Sports Medicine, 2011

9. Gu JQ, Shan YH : Therapeutic effect of triple Puncture at Tianzong (SI 11) as main method on obstinate tennis elbow. (Shanghai Guanghua Hospital of Integrated Traditional Chinese and Western Medicine, Shanghai 2000)

10. Hussien A. Heshmat\& Nader Shalaby: The physiology of muscular fatigue (Publishing book center - Cairo, 2003)

11. J. H. Cyriax: the pathology and treatment of tennis elbow, The Journal of Bone \& Joint Surgery, Volume 18, Issue 4, 2003

12. Jean-Louis Croisier, Marguerite FoidartDessalle, France Tinat, Jean-Michel Crielaard and BenedicteForthomme: An isokinetic eccentric Programme for the management of chronic Lateral epicondylar. (Department of Physical Medicine and Rehabilitation, Belgium, 2006)

13. Jeng-Ming Tsai: Sport injuries of upper extremity in tennis players (2011)

14. Julia Williams: Tennis elbow clinical guide (Osteopath• www.juliawilliams.co.uk Feb 2009)

15. Leanne Bisset, Brooke Coombes, and Bill Vicenzino: tennis elbow clinical evidence (C) BMJ Publishing Group Ltd 2009)

16. Majima T. Yasuaa: Rehabilitation after hamstring anterior cruciate Ligament reconstruction (CLIM orthop, Vo 397 PP360 $\mathrm{Ph}$ 2002)

17. McKay AB: Tennis Elbow Everywhere. (Australian Musculoskeletal Med. November 2005, 10:127 - 130)

18. Pierre Rouzier: Epicondylitis (Tennis Elbow) (McKesson Health Solutions LLC. 2003)

19. Shmidt N, Van Der Windt D.: Tennis elbow in Primary care. (C) BMJ 2006 : 333 : 927 - 928)

20. Shoulder Doc. UK: Tennis Elbow (www.shoulderdoc.co.uk 2011)

21. Smibert Chambler: Tennis Elbow' or lateral Epicondylitis, NHS foundation, 2009

22. Thomas G. Wadsworth: Tennis elbow: conservative, surgical, and manipulative Treatment (British medical journal, edition 294, 1987)

23. UsamaRiad: Sports Medicine and physical therapy (Publishing book center - Cairo, 2001)

24. Van Den ende et al: Dynamic exercise therapy in rheumatoid arthritis. (British Journal of rheumatology, 37 (6) : 677-87. 1998)

25. Werner RA, Franzblau A, Gell N, Hartigan A, Ebersole M, Armstrong TJ. : Predictors of Persistent elbow tendonitis among auto assembly workers, (J Occup.Rehabil, 2005, 15 : $396-4$ 
AlaaEldin Mohamed Elewa, Yasser Kamal Ghoniem, Mohamed Kamal Moussa 